CAHIERS DE

NARRATOLOGIE

\section{Cahiers de Narratologie}

Analyse et théorie narratives

10.2 | 2001

La voix narrative

\title{
Les voix de l'homme et de l'ombre dans Quand cinq ans seront passés de Federico García Lorca
}

\section{Gérard Lavergne}

\section{(2) OpenEdition}

1 Journals

Édition électronique

URL : http://journals.openedition.org/narratologie/10207

DOI : 10.4000/narratologie. 10207

ISSN : 1765-307X

Éditeur

LIRCES

\section{Édition imprimée}

Date de publication : 1 janvier 2001

Pagination : 155-184

ISBN : 2914561032

ISSN : 0993-8516

\section{Référence électronique}

Gérard Lavergne, «Les voix de l'homme et de l'ombre dans Quand cinq ans seront passés de Federico García Lorca », Cahiers de Narratologie [En ligne], 10.2 | 2001, mis en ligne le 01 janvier 2001, consulté le 11 juin 2020. URL : http://journals.openedition.org/narratologie/10207 ; DOI : https://doi.org/ 10.4000/narratologie.10207 


\title{
LES VOIX DE L'HOMME ET DE L'OMBRE DANS QUAND CINQ ANS SERONT PASSÉS DE FEDERICO GARCÍA LORCA.*
}

\author{
Gérard LAVERGNE \\ Université de Nice-Sophia Antipolis
}

F. Garcia Lorca, évoquant le théâtre de la fin du XIXe et du début du $\mathrm{XX}^{\mathrm{e}}$ siècles, parle d'un teatro al aire libre (Théâtre à l'air libre), qu'il oppose au teatro bajo la arena (théâtre sous le sable).

Qu'appelle-t-il théâtre à l'air libre ? Prenons un exemple :

Jacinto Benavente (1806-1954), contemporain de Lorca, est un auteur à succès du teatro al aire libre. Son théâtre fait la chronique d'une bourgeoisie qui va au spectacle pour se divertir. Celle-ci n'est pas intéressée par les problèmes sociaux ni moraux qui pourraient la gêner et même l'offenser. Lorsque Benavente présente "El nido ajeno » (le nid d'autrui) en 1894, où il s'interroge sur l'éventualité qu'un de ses personnages de mère puisse être infidèle et puisse disposer d'elle-même, il connaît un échec total. Il échoue donc quand il critique les certitudes de cette classe, et ses valeurs morales.

Par contre, avec «Los intereses creados» (Les intérêts suscités) en 1909, il connaît un énorme succès. Le thème ? Crispin, valet et ami du désargenté Léandre, monte une intrigue qui va permettre à ce dernier d'épouser la jeune Sylvia, uniquement parce qu'elle est riche... L'intrigue et le mensonge initial sont découverts, mais l'amour qu'éprouve déjà Sylvia naît aussi dans le cœur du prétendant. L'amour et le pardon justifient le mariage entre les jeunes-gens.

* Cette communication, ici revue et amplifiée pour un lecteur non hispaniste, a été présentée lors du Colloque sur Lorca organisé par l'Université de Poitiers en janvier 1999. Elle a été publiée dans Lorca. L'écriture sous le sable. Édit. Thibaudeau, Poitiers, La Licorne, 1999. 
Avec le «théâtre à l'air libre » nous avons donc un théâtre de mœurs et de salon, s'appuyant sur un décor réaliste de carton pâte.

A ce théâtre du politiquement correct bourgeois de la fin du XIX ${ }^{\mathrm{e}}$ et début du XXe $\mathrm{X}^{\mathrm{e}}$, Lorca oppose le " Théâtre sous le sable »; un théâtre qui montre violemment et en toute liberté ce qui se passe quand l'être humain, enlevant son masque, laisse s'exprimer ses pensées les plus profondes, ses sentiments, les désirs qui le poussent, et qu'il doit dissimuler à son prochain pour ne pas être condamné par la société. C'est le théâtre de la vie dans ses aspects les plus inconscients et les plus contrôlés par les forces diverses qui empêchent l'expression d'une véritable identité.

Dans le cadre de cette communication, une évocation rapide du théâtre de Lorca nous semble indispensable.

1920 El maleficio de la mariposa (le maléfice de la phalène) : le jeune cafard, le poète Cafardet, rejette un monde de conventions sociales. Amoureux d'un papillon blessé, symbole d'un rêve, d'une vision idéale éloignée de celle du milieu où il vit, il meurt. La pièce est un échec complet : le public n'y comprend rien.

1928 El amor de Don Perlimplín con Belisa en su jardín (Les amours de Don Perlimplin avec Bélise dans leur jardin) : Un vieillard épouse Bélise, grâce à deux entremetteuses (une bonne et une mère). C'est un mariage social et d'intérêt. Il a été séduit par la jeune femme qu'il a vue nue par le trou d'une serrure. Elle le trompe le soir de ses noces, dans sa soif d'amour, avec 5 représentants des cinq races. Lui se suicidera. La pièce a été interdite par la censure.

1930 La zapatera prodigiosa (La savetière prodigieuse) : un vieillard, pour fuir la solitude, épouse une jeune femme qui lui reste fidèle mais, se sentant aliénée, le dispute tout le temps lorsqu'il est là. Il s'en va.

Elle le sublimise en son absence et quand il revient elle se hâte de le disputer à nouveau. Guerre des sexes ? Peut-être. Vision de l'incompréhension entre les êtres sans aucun doute.

1930 El Público (Le Public) Le thème de l'homosexualité y est clairement traité : Roméo et Juliette dans la pièce sont deux hommes. 
Les voix de l'homme et de l'ombre dans

Quand cinq ans seront passés de F. García Lorca

1931 Así que pasen cinco años (Quand cinq ans seront passés)

1933 Bodas de sangre (Noces de sang) La fiancée, amoureuse d'un autre homme épouse son fiancé par obligation. Mais après la noce, la fiancée se sauve avec son amant. C'est la force du sexe a dit Lorca. Les deux hommes se tuent. C'est l'amour et l'instinct dominés et réprimés par la société.

1934 Yerma est le drame de la femme inféconde, de la libido frustrée et de l'obsession de la maternité.

1935 Doña Rosita la soltera o el lenguaje de las flores (Doña Rosita la vieille fille ou le langage des fleurs). Une jeune-fille abandonnée par son fiancé vieillit seule et vit le drame de la célibataire en Espagne, agressée par la société.

1936 La casa de Bernarda Alba (La maison de Bernarda Alba). La volonté d'une mère soumet à la règle sociale ses filles frustrées, maintenues enfermées dans un espace clos, aussi réaliste que métaphorique. Adela, l'une d'elles se rebelle, et après avoir connu l'amour, se suicide.

Spectacle du conflit des pulsions les plus profondes, nous voyons pourquoi la thématique lorquienne ne convenait pas au public bourgeois du «théâtre à l'air libre ».

En 1933 Lorca, au cours d'un interview en Amérique Latine parle de deux de ses pièces. La première, écrite en prose et en vers, il n'a pas l'intention de la créer à Buenos Aires. Il s'agit de Así que pasen cinco años : Lorsque cinq ans seront passés ${ }^{1}$ un mystère sur le temps. La seconde, El Público, il n'a pas l'intention de la créer, ni à Buenos Aires ni ailleurs, car, dit-il, "Je pense qu'il n'y a pas de troupe qui osera la représenter, ni de public qui la tolèrera sans s'indigner $»^{2}$.

${ }^{1}$ Les pages que nous indiquons sont celles de l'édition en espagnol recommandée par le jury des concours 1999: Así que pasen cinco años. Leyenda del tiempo : Margarita Ucelay, Madrid, Catedra n. 597. Il existe une traduction de Así que pasen cinco años: Lorsque cinq ans seront passés de Marcelle Auclair : dans Federico García Lorca, CEuvres complètes, Paris, 1990, Gallimard, La Pléiade T. II, p. 385440.

Nous évoquons l'œuvre dans notre texte par Quand cinq ans ...

2 Federico García Lorca (FGL) 0.C. Madrid, Aguilar, 1965, p. 1731. 
En 1936, à Madrid, il déclare ces pièces, en particulier Quand cinq ans... injouables, et ajoute : « dans ces pièces impossibles se trouve ce que j'ai vraiment voulu faire ${ }^{3}$.

Pourquoi pièces impossibles? La réponse nous est donnée par le poète lui même. En 1934 il affirmait déjà son intention d'aborder des «thèmes et des problèmes que les gens ont peur d'aborder $»^{4}$; car pour lui, dit-il en juillet 1936, le théâtre " poésie qui sort du livre et se fait humaine $»^{5}$ réclame que les personnages qui y apparaissent soient «si humains, si horriblement tragiques et liés à la vie et au moment avec une telle force, qu'ils montrent leurs traîtrises, que l'on apprécie leurs souffrances et que sorte sur leurs lèvres tout le courage de leurs paroles pleines d'amour et de dégoûts ${ }^{6}$.

En avril 1936 Lorca annonce le début des répétitions de Quand cinq ans seront passés, Légende du temps, pièce en trois actes et quatre tableaux qu'il avait terminée en 1931.

Paradoxe que de parler de narration pour une œuvre théâtrale, parler de diégésis alors que nous sommes en mimésis. Et pourtant, que de signes se font voix dans Quand cinq ans... pour crier au spectateur un drame auquel il assiste et qui est peut-être, dans son espace et dans son esprit, le sien.

Nous prendrons pour cette communication la voie tracée par Gérard Genette. Ce dernier, dans son Discours du récit ${ }^{7}$, à propos de l'instance narrative, parle de la voix. Il cite en particulier un sémiologue, Vendryes, pour qui celle-ci est "l'aspect de l'action verbale considérée dans ses rapports avec le sujet »; et Genette d'ajouter : ce sujet n'est pas seulement celui qui accomplit ou subit l'action, mais aussi celui (le même ou un autre) qui la rapporte, et éventuellement tous ceux qui participent, fût-ce passivement, à cette activité narrative.

Dans une œuvre théâtrale, tout participe activement ou non à cette activité narrative ; car tout $\mathrm{y}$ " parle, tout a un sens et donne un sens. Nous parlerons donc des voix des

${ }^{3}$ Idem, p. 1811.

4 Idem, p. 1767.

5 Idem, p. 1810.

6 Idem, p. 1810.

${ }^{7}$ Gérard GENETTE : Figures III, Paris, Seuil, 1973, p. 226. 
cyberacteurs, qu'ils soient animés ou inanimés $»^{8}$. Après avoir résumé quand cinq ans..., nous essaierons de déterminer les différentes voix d'un texte qui présentent l'homme sur le théâtre du monde et nous verrons que cette pièce que l'on a dite philosophique, sociale, auto sacramental, jeu de rôles... évoque en définitive la métaphysique d'un homme livré à l'ambiguïté de sa personne sociale et aux forces obscures qui l'agitent et le tuent. C'est la raison pour laquelle nous parlerons des voix de l'Homme et de l'Ombre.

L'argument : Acte I : la Dactylo aime le Jeune Homme, qui ne l'aime pas. Il aime la Fiancée qu'il doit épouser dans cinq ans.

Acte II : Lors de leur rencontre il s'avère que celle-ci ne l'aime plus. Elle lui conseille d'aller retrouver celle qui l'aimait.

Acte III : Il retrouve la dactylo, mais elle lui demande maintenant d'attendre cinq ans. Il meurt.

C'est ce que nous appellerons la fiction'.

Un sinopsis plus complet laissera vite apparaître une pièce d'accès difficile, complexe, règne de l'uchronie et de l'atopie, à portée universelle, comme l'indiquent les noms génériques.

8 Tout signe s'appuie sur un référent et sa narraticité fait sens. Celui-ci dépend de la culture du destinataire amené à interpréter sa valeur symbolique, quand ce n'est pas son aspect psychanalytique. Lorca dans cette pièce sollicite à tout instant l'imaginaire et la symbolique du spectateur, jouant avec les bruits, les couleurs, les êtres et les choses.

L'espace réservé à ce travail ne nous permettra pas d'interpréter toute la symbolique de Lorca, couleurs, mise en scène, etc, ni les aspects psychiatriques de ses œuvres, ce qui serait hors de ses limites et surtout de nos compétences. Nous ne relèverons que quelques exemples particulièrement aptes à éclairer notre propos. C'est, à ce sujet, ce que nous appellerons les voix narratives de l'Ombre ou de l'inconscient.

${ }^{9}$ Ce résumé sera considéré comme celui de la fiction de Dans cinq ans... Cette chaîne événementielle sera dite le récit premier. Le Jeune Homme en est le personnage principal, les autres personnages seront des personnages et acteurs seconds, émetteurs de leur propre symbolique. Souvent reflets d'un autre symbole, ils présentent leur propre point de vue, lui-même méandre du flux de la conscience du personnage principal dans un discours de récit second. 
Acte I : dans l'espace fermé de la bibliothèque, nous surprenons la conversation du Jeune Homme en pyjama et d'un Vieillard. Celui-ci, mentor et projection du premier dans le futur, veut l'empêcher d'agir pour le protéger de l'usure du temps. Ils sont assis.

La Dactylo traverse la scène. Le héros ordonne de fermer la fenêtre. Les deux personnages évoquent le mariage du Jeune Homme avec la Fiancée partie pour un long voyage. Le Vieillard demande si le Père est venu. Le Jeune Homme répond : «Jamais! pour le moment ce n'est pas possible/.../ tant que cinq ans ne seront pas passés » (p. 196).

La dactylo reparaît et déclare qu'elle veut partir. Elle l'aime mais il ne la retient pas.

Arrive le premier Ami qui prend la vie et les femmes à bras-le-corps et l'invite à en faire autant.

Intervient alors une scène du plus grand lyrisme onirique : une chatte et un enfant morts entrent en scène et chantent leur regret de la vie en attendant d'être enterrés. Un domestique apprend aux personnages que le fils de la concierge vient de mourir et qu'un chat a été lapidé.

Arrive le $2^{\mathrm{e}}$ Ami qui s'installe dans la pièce.

La dactylo repasse ; à ses questions le Jeune Homme répond qu'il n'a pas besoin d'elle. Fin du premier acte.

Acte II : Une chambre 1900 ; une fenêtre ouverte sur le balcon : la chambre de la fiancée. Celle-ci saute de son lit en peignoir de dentelle; elle fait entrer par la fenêtre un rugbyman américain en tenue. Dans ses bras elle lui crie sa passion. Lui, muet, fume sans arrêt.

A l'atmosphère onirique du $1^{\mathrm{er}}$ acte succède une impression de réalisme auquel contribue le dialogue entre la Domestique, charmée par les manières délicates du Jeune Homme, et la fiancée qui ne veut plus de lui.

Le Père, plus intéressé par une éclipse de lune ${ }^{10}$ que par sa fille, est navré d'une décision qui va à l'encontre des règles conventionnelles.

10 Voir l'article de Nadine LY sur l'éclipse in Grammaire et dramaturgie du temps dans Así que pasen cinco años, Les Langues Néo-Latines, Concours, 4e trimestre 1998, n 307, p. 133. 
Le fiancé va venir, le délai de cinq ans est achevé. Le Jeune Homme arrive au rendez-vous. La Fiancée le congédie, l'invite à aller prendre femme ailleurs et part avec le joueur de rugby.

La fiancée disparue, entre en scène un Mannequin portant la robe de mariée.

Un dialogue onirique s'instaure, le Mannequin reproche au Jeune Homme son manque de résolution et le pousse à aller chercher la Dactylo qui l'aimait. Ce dernier promet de la ramener avant la fin de l'éclipse.

Acte III :

$1^{\text {er }}$ tableau : le spectateur se trouve devant un bois, de grands troncs. Au centre, surélevé, un castelet entouré de rideaux baroques. Un petit escalier le relie au praticable. Quand le rideau se lève, deux silhouettes glissent entre les troncs, le visage et les mains d'un blanc de plâtre.

Arlequin entre, vêtu de noir et de vert, les deux mains derrière le dos.

Il évolue comme un danseur, déclamant un poème sur l'antinomie entre le temps et le rêve.

Une Jeune Fille paraît. Elle évoque un fiancé qui l'attend au fond de la mer. Arlequin va le lui faire retrouver. Elle n'y croit pas. Il appelle l'Homme. Un clown, son allégorie apparaît. Il salue le public. Arlequin lui demande de donner à la Jeune Fille son fiancé, un homme sublimisé par les cinq ans passés, un fantasme dans un monde féerique. Effrayée par la réalisation d'un mythe qu'elle sent proche, ce qui l'en priverait, la Jeune Fille refuse. Elle sort.

On entend des cors de chasse. Entrent la Dactylo en tenue de tennis et une femme en jaune. La Dactylo métamorphosée voit en celle-ci son propre double et la chimère que son inconscient a forgée au cours des cinq années d'attente pour être aimée.

Le Jeune Homme apparaît en costume sport, venu directement de la fin de l'acte II. Il cherche celle qui l'a aimé. Il la veut à présent pour assurer sa paternité. Le spectre de l'enfant mort passe devant les deux personnages.

Mais il faut à la Dactylo le temps de s'habituer à cette nouvelle situation. Elle se donnera à lui ... quand cinq ans seront passés. Fin du tableau I. 
$2^{\text {e }}$ tableau : bureau de l'Acte I. Contraste total avec la vision baroque de la chambre de la Fiancée à l'Acte II. Des valises ouvertes, la robe de mariée sur un mannequin, une table de jeu.

Le Domestique et la Femme de chambre commentent la mort du fils de la concierge et du comte italien. Le Jeune Homme entre. Le Domestique lui demande s'il s'est reposé après son voyage. Ils attendent trois visiteurs. Une partie de cartes est organisée. Le jeune homme est obligé de jeter sur la table celle qu'il n'a jamais voulu utiliser: son as de cœur. Les visiteurs l'ont obtenue. Ils s'éloignent. Le héros agonise et meurt.

Nous avons donc dans Quand cinq ans...une chronologie traditionnelle : exposition, développement, dénouement.

Le schéma fictionnel pose le problème de l'homme face au Temps et face à lui-même. Lorca va répondre à cette problématique en fonction de sa propre problématique.

Le temps dans la pièce est donné en premier lieu par des informations mathématiques. En effet, au lever du rideau du premier acte, nous trouvons conversant, assis, le Jeune Homme en pyjama bleu" et le Vieillard en jaquette grise : il est six heures. Nous en sommes d'autant plus convaincus que l'informant est annoncé trois fois d'entrée par des voix différentes. Une didascalie signale qu' " une horloge sonne six heures » (p. 194)

Le Vieillard reprend l'information : "Six heures », et le Jeune Homme en fait de même, donnant d'autres éléments prémonitoires : "Oui, six heures et une chaleur excessive (il se lève). Il y a un beau ciel d'orage. Plein de nuages gris » (p. 195)

$\mathrm{Au}$ cours de leur conversation nous savons que la Fiancée est partie en voyage avec son père et qu'il reviendra quand cinq ans seront passés (p. 196).

La fiancée a coupé ses nattes ; le Jeune Homme est sûr qu'elle les récupérera au cours de ces cinq années (p. 200) ${ }^{12}$. A la fin de l'acte, le Jeune Homme demande à son valet : Juan /.../

11 J. CHEVAliER, A. GHEERBRANT : Dictionnaire des symboles, Paris, Laffont, Jupiter, 1982.

12 Voir les observations de Nadine LY sur le chiffre cinq dans son article cité, note 10, p. 150 . 
Quelle heure est-il ? - Six heures exactement Monsieur.» (p. 243)

Le temps n'a pas avancé.

Acte II. La Domestique de la Fiancée évoque avec elle le Jeune Homme : «Un homme si aimable. Qui vous a attendue si longtemps /.../ Cinq ans. » (p. 254).

Le père de la Fiancée, scandalisé par la volonté de sa fille de refuser le mari programmé, rappelle que la promesse de cette union a été faite « il y a cinq ans, jour pour jour. » (p. 264).

Acte III, $2^{\mathrm{e}}$ tableau : A nouveau dans le bureau de l'acte I, le valet demande au Jeune Homme s'il s'est assez reposé après son voyage. On peut imaginer qu'il a fait un déplacement pour retrouver la Fiancée après cinq ans d'attente ou qu'il se réveille. Et la pièce se termine sur une dernière information : l'horloge sonne douze heures : Minuit...

Ces éléments ne manquent pas de poser problème et de désarçonner le spectateur ou le lecteur à la première réception de l'œuvre. En effet, dès le premier acte, alors que le Jeune Homme, le Vieillard et l'Ami discutent, le discours de ce dernier est coupé et le paratexte didascalique indique qu'ils vont se cacher derrière un paravent brodé d'étoiles. Ils sont écartés de ce que va suivre le spectateur, et un récit second envahit le récit premier:

Le Chat, qui est une Chatte, et l'enfant apparaissent. Ils parlent.

Ils sont morts ; elle, tuée par des garnements, lui, d'une crise cardiaque.

Ils vont être enterrés et font tout pour reculer l'échéance, sans succès.

La luminosité passée au bleu à leur entrée en scène (p. 217) retrouve ensuite sa couleur normale (p. 230). La fiction reprend son cours. le Domestique entre annoncer à son maître ce que le spectateur vient d'apprendre : la mort des deux acteurs.

Remarquons que nous avons là une séquence analeptique, donc un temps déjà écoulé au moment du décrochage du temps du récit premier et un espace virtuel qui envahit celui réel de ce récit. Cette anachronie inexpliquée est maintenant bien présente dans l'esprit du spectateur; il n'en verra la valeur dramatique et son aspect proleptique qu'à l'acte III. 
Mais pour le moment il reste perplexe. Il le sera davantage quand, à la fin de ce même acte I, Juan, le valet, interrogé sur l'heure par son maître répond (avec une intention marquée) " exactement six heures monsieur» (p. 243), et « le Jeune Homme de taper doucement avec ses doigts sur la table " (p. 244), la gestuelle traduisant sa préoccupation.

A l'acte II nous l'avons vu, le continuum fictionnel est normal. Les cinq ans annoncés dans l'acte I se sont bien réalisés. Le temps a fait son œuvre chez la Fiancée devenue femme.

A l'acte III ler tableau, le Jeune Homme poussé par son désir d'avoir un enfant et d'assurer son éternité, parti solliciter la Dactylo, échoue.

$\mathrm{Au} 2^{\mathrm{e}}$ tableau, de retour dans la bibliothèque, il meurt d'avoir joué son cœur. La ronde des heures, de ses heures, est achevée. L'horloge sonne minuit. Le temps de la fiction est donc de six heures au plus ${ }^{13}$.

Quelles seront les réactions du spectateur quand le rideau tombera ? A l'acte I il assiste à l'intrusion d'un passé, vécu comme un présent, dans le présent du récit premier, le présent du héros. L'existence du passé ainsi revisité en mimésis est donc niée. Le présent de la fiction est nié également, puisqu'à la fin de cet acte il est toujours six heures.

Le futur est annoncé par le Jeune Homme : Il attendra cinq ans ; mais à l'acte II il est lui aussi nié, puisqu'après le soidisant espace temporel de cinq ans d'attente, confirmé par plusieurs voix (la servante p. 254 , le père p. 261 et 264 ), le Jeune Homme arrive chez la Fiancée, déclare que le voyage a été très long et s'excuse de son retard, car il vient de corriger

13 Des études avancent que les douze coups peuvent indiquer six heures car certaines horloges redoublent les heures qu'elles sonnent. Sans doute, mais alors pourquoi parler de six heures à l'acte I quand une horloge sonne six heures sans spécifier le nombre de coups? Nous en resterons au texte. Les douze coups sonnent à notre avis minuit. Le temps de la fiction est de six heures: de $6 \mathrm{~h}$ aux douze coups de minuit. Le temps de la narration durera le temps exact de la représentation, jusqu'à la fin du paratexte didascalique (...El reloj da las doce), (p. 353). Voir à ce sujet la conclusion de l'article de $\mathrm{P}$. Thiollière dans Le théâtre impossible de Lorca. El público, Así que pasen cinco años. Textes réunis par Michèle Ramond, Editions du Temps ; Paris, 1998, p. 139. 
des enfants qui lapidaient une chatte (p. 265). Il est donc un peu plus de 6 heures à peine.

Le futur est nié également à l'acte III, 2e tableau, au début duquel la servante et le domestique (p. 334) commentent l'enterrement de l'enfant mort, annoncé à l'acte I, avant que le valet ne demande à son maître s'il est bien reposé de son voyage, pour voir la Fiancée sans doute (p. 336). Or, à la fin de ce tableau, l'horloge sonne les douze coups de minuit, informant qui indique le temps biologique du héros depuis le début de la fiction. Le seul temps objectif est donc bien de 6 heures.

Le spectateur, impliqué, interpellé directement, nous le signalons, dans la pièce par le «Bonsoir» du clown (p. 298) et son "Bonjour» (p. 302), ne pourra que constater que le temps éclaté qu'il vient de vivre est une façon de nier le Temps dans la représentation uchronique qui en est faite, et de montrer que seul compte en définitive la durée, le temps vécu, indestructible, conservé en son cœur par le personnage, en train de rêver une vie qui l'emporte irrémédiablement vers le néant.

Car ce Jeune Homme qui n'a pas de nom, et par suite n'est qu'une métaphore de notre ÊTRE, ce Jeune Homme rêve. Dans le flot de sa conscience, par la voix (e) du monologue intérieur qui se fait la voix de l'Homme, il raconte ses angoisses, les angoisses de l'Humanité, de son humanité. Être de vie, il refuse le Temps qui est la mort, et il clame son désir d'éternité.

C'est ainsi que Quand cinq ans... se fait symphonie à la vie, même si l'Homme en fait un chant à la mort.

Le prélude en est la discussion entre le Jeune Homme et le Vieillard, un de ses nombreux doubles. Ce dernier pousse son interlocuteur à refuser l'action qui use et précipite vers le néant. Aussi ne se veut-il que futur, même d'un passé non réalisé, futur souvenir donc, et exulte-t-il quand il entend le héros affirmer :

"Je ne veux pas d'herbe chez moi, ni de meubles abîmés /.../ mais des choses vivantes qui bouillonnent dans leur sang, et dont tous les profils sont intacts. " (p. 193) 
Le Vieillard acquiesce avec une phrase sybilline qui le trahit : «Très bien! C'est-à-dire (baissant la voix) il faut se souvenir, mais se souvenir avant. » (p. 194)

Il ouvre au Jeune Homme une perspective : le futur, et, nous le constatons, refuse de prendre en considération le temps présent. Rappelons que les annotations le montrent avec de grandes lunettes d'or, et cette voix narrative aussi nous invite à voir en lui le symbole du futur du jeune héros dans son devenir déjà échu. Pour éviter à ce dernier l'état de décrépitude, il l'encourage fortement à suivre la route qu'il s'est déjà tracée : ne rien faire, attendre, car agir use et détruit le fil de vie que les Parques ont prévu pour l'homme. Il l'invite à rêver et à garder dans son cœur les souvenirs qui eux, ne s'usent pas :

« Jeune Homme : je gardais mes bonbons pour les manger plus tard.

Vieillard : Plus tard, n'est-ce pas ? Ils sont bien meilleurs. Moi aussi » (p. 192)

Tout au long de la pièce ce personnage suivra les pas du héros, et l'abandonnera aux mains des Parques au dernier tableau, après avoir tout fait pour le figer et le persuader du bienfait de vivre enfermé dans son bureau et en lui-même.

Un autre double, l'Ami, entre (bruyamment) ; le Vieillard s'en va. Le Jeune Homme en pleine introspection, enfermé dans son espace clos, sera obligé d'entendre cette nouvelle voix. Les interventions de l'Ami sont significatives :

"Quel silence dans cette maison!» (p. 209)

«Un homme seul, un homme sérieux, avec cette chaleur!" (p. 210)

«Et où sont les portraits des filles avec qui tu couches?»(p. 210)

« Je vais te jeter à la rue à coup de canne. » (p. 211)

« Hier j'ai fais trois conquêtes, et comme avant-hier j'en ai fait deux et aujourd'hui une,... Eh bien en définitive... je n'en ai pas une, car je n'ai pas le temps. » (p. 211)

Cette voix est celle du présent, éphémère, qui n'existe que pour disparaître, celle du refus de l'attente p. 211, celle de l'action devenue agitation échevelée et aussi inutile que l'attente du Jeune Homme. Les didascalies nous présentent le personnage comme très agité, très joueur avec le héros, se 
voulant persuasif mais sans résultat. Le jeune Homme répond le plus souvent «Laisse-moi !» (p. 210) et il refuse de sortir. Après l'incursion analeptique dans l'au-delà avec la scène de la chatte et de l'enfant, apparaît soudain, par la porte de gauche - porte de l'inconscient et du rêve - le $2 \mathrm{e}$ ami, de blanc vêtu, avec d'énormes boutons bleus; les couleurs de la pureté et du rêve. Le texte précise que ce rôle peut être tenu par un très jeune acteur ou une jeune fille. Lui ne sait guère par où il est entré : "Par n'importe où. Par la fenêtre. » (p. 235) dit-il ; et il annonce : "Un orage va éclater... mais un bel orage celui qui est tombé l'année dernière. » (p. 235)

Il dresse ainsi un décor sonore, métaphore en quelque sorte de l'orage qui agite le Jeune Homme et qui l'emportera. Miroir, double du héros, il refuse comme lui le passage du temps, son flux vers l'avenir et l'échéance finale : la mer, la mort. Il évoque les gouttes de pluie qu'il a vues tomber et les petites femmes nues qu'elles renfermaient (p. 236), et il s'adresse aux autres personnages sur la scène. " $\mathrm{Ne}$ les avez-vous pas vues ? Lorsque j'avais cinq ans... non quand j'en avais deux... Je mens ! un an, un an seulement. » (p. 236)

Il se rappelle celle qu'il avait gardée deux jours et qui n'avait pas grandi car «Elle s'est faite de plus en plus petite, plus enfant, comme il se doit, comme il est juste jusqu'à ce qu'il ne reste plus d'elle qu'une goutte d'eau. » (p. 236)

Le spectateur aura compris combien l'idée de régression domine l'esprit du personnage qui révèle sa volonté de ne pas vieillir et de remonter le temps ; combien son inconscient est orienté non vers le futur mais vers le passé, combien il est un des aspects du héros lui-même qui refuse d'agir et voudrait être ce qu'il a été et ainsi conserver son intégrité. Son discours s'avère être un discours de mort où se multiplient les avertissements.

«Avant d'entrer ici, j'ai vu un enfant qu'on allait enterrer avec les premières gouttes de pluie. Voilà comment je veux que l'on m'enterre moi. Dans un cercueil petit comme ça. » (p. 238)

Au Vieillard qui l'a traité de fou il rétorque : «Fou, parce que je refuse d'être comme vous tout douleurs et rides. » (p. 237) En fait il rejoint le Vieillard qui conseille au Jeune Homme de ne rien faire, mais il en montre les conséquences, ce que ce 
dernier ne fait pas, et il affirme : "Il n'y a qu'à réveiller les choses doucement. »

Il ajoute : «Par contre dans quatre à cinq ans il existe un puits dans lequel nous tomberons tous. » (p. 240) $)^{14}$

Le 1er Ami révèle le sens profond de la conversation : «Tout cela n'est que la peur de la mort. » (p. 237)

A la fin de l'acte, le héros refuse l'amour de la Dactylo qui passe devant lui : il n'a rien compris au message de l'Ami.

L'acte se termine sur une complainte que chante en s'assoupissant le 2e ami :

«Je reviens prendre mes ailes,

laissez-moi m'en retourner.

Je veux mourir en étant

source.

Je veux mourir loin

de la mer. » (p. 243)

Le Jeune Homme rêve.

Lorca, nous le voyons, fait passer le spectateur derrière la psyché du moi et entendre les diverses voix de la conscience du héros. En focalisation interne, nous écoutons, avec le personnage éclaté, le Vieillard, l'Ami, le 2e Ami. Ce sont autant de cyberacteurs d'un jeu de rôles où chacun présente les diverses attitudes possibles de l'homme devant le problème de la vie. En fait, allégories d'un auto sacramental, ils sont, rêvées par le Jeune Homme, les voix d'une parabole sur l'homme en situation dans le monde où le temps passe, l'use et l'entraîne vers le néant, et qui doit répondre à la question: Que faire pour lutter contre ce passage inexorable, que faire pour durer? attendre ou agir?

Le héros a choisi d'attendre cinq ans pour se déclarer à sa fiancée.

Acte II. Contraste saisissant avec le bureau austère du Jeune Homme. La didascalie annonce

«Une chambre à coucher 1900. Des meubles étranges. De grands rideaux à plis et à glands /.../ Au milieu un lit

14 Il a été remarqué combien ces mots avaient un caractère prémonitoire. La pièce est donnée comme achevée le 19 août 1931. Le 18 juillet 1936 éclate la Guerre Civile. Lorca meurt assassiné le 19 août 1936. 
aux draperies abondantes et beaucoup de plumes. A gauche une coiffeuse portée par des anges qui tiennent en main des bouquets de lampes électriques. Le clair de lune entre par les balcons ouverts. On entend le claxon furieux d'une automobile. La fiancée saute de son lit dans une splendide robe de chambre couverte de dentelles et d'énormes nœuds roses /.../ Ses cheveux sont très bouclés. » (p. 245)

La narraticité du décor, sa voix en quelque sorte, suggère les courbes gracieuses, la vivacité, la sensualité érotique de la Fiancée qui réagit dès qu'elle entend le klaxon du Joueur de rugby. Les balcons, par où pénètre le clair de lune, laissent imaginer sa soif d'évasion mais aussi sa tendance au rêve. C'est par le balcon qu'entre le rugbyman. Celui-ci, en tenue, est la bestialité métaphorisée, l'archétype de la virilité érotomaniaque. Il serre dans ses bras la jeune fille qui ne semble du reste pas l'intéresser, et il fume cigare après cigare, symboles phalliques de ses nombreuses conquêtes.

L'une d'elle est la Fiancée qui, dans ses bras, regrette son absence de la veille et signale à celui qu'elle appelle son dragon (p. 246), avoir vu le cheval blanc aux sabots d'or.

L'évocation du cheval par la Fiancée, symbole érotique du désir, fait d'elle l'archétype de la femme désireuse d'assumer sa vie passionnelle et de s'opposer aux contraintes sociales ou morales d'un monde qui l'oppresse, même si un tel comportement implique des risques, puisque, nous le rappellerons, le dragon est le symbole, entre autres, soit d'un gardien sévère, soit du mal et des tendances démoniaques ${ }^{15}$. Le rugbyman sorti par le balcon, la Fiancée et la servante évoquent le Jeune Homme dont les bonnes manières ont séduit cette dernière. Le héros entre dans la pièce où la

15 Pour certains psychanalystes, passé le seuil de la puberté, le cheval devient pleinement le symbole de l'impétuosité du désir de la jeunesse de l'homme, avec tout ce qu'elle contient de fécondité, de générosité. Dans la poétique de Lorca, le cheval a une signification indubitablement érotique.

Le lecteur aura noté que nous parlons de symbole mais pas de surréalisme. L'écriture de Lorca dans cette pièce ne montre en particulier aucune trace de ce qui en fait la caractéristique : l'écriture automatique, bien au contraire. 
Fiancée, en tailleur strict, se prépare à se sauver pour rejoindre son séducteur. Le changement de son costume est signe lui-même. Interpellant le Jeune Homme, lui demandant s'il avait les qualités qu'elle attribue à son amant : "Et toi, tu ne jouais pas au rugby ? » (p. 267) elle fait le portrait de l'être que ses vingt ans l'ont amenée a préférer ${ }^{16}$. Elle repousse celui que les conventions sociales lui avaient proposé. Ce dernier se rebelle :

«Jeune Homme: Désormais personne ne pourra séparer mes bras de ton cou !

Fiancée : Ce ne sont pas tes bras, ce sont les miens. C'est moi qui veux me brûler à un autre feu.

Jeune Homme: Il n'y a de feu que le mien. (il l'embrasse) parce que je t'ai attendue et maintenant je gagne mon rêve. » (p. 268)

«Rêve » est le mot qu'il ne devait pas prononcer lui fait remarquer la Fiancée car, dit-elle "Ici on ne rêve pas. Moi je ne veux pas rêver. » (p. 269)

Elle le pousse à aller chercher une femme à qui il pourrait faire des tresses, symbole d'asservissement et de vie sans issue. Celui-ci, comme s'il se réveillait (p. 269), réagit faiblement («Non») Elle lui décoche les flêches finales qui devraient mettre en furie tout fiancé délaissé :

« Comment vais-je te laisser entrer dans ma chambre quand un autre y est déjà entré ? /.../

Deux jours seulement ont suffi pour me sentir chargée de chaînes. Dans les miroirs et parmi les dentelles de mon lit j'entends déjà le gémissement d'un enfant qui me poursuit. »(p. 270)

Nous arrivons ici au climax de la pièce. Là en effet le Jeune Homme prend conscience de son comportement anormal et le mot enfant devient la voix de son subconscient qui l'interpelle et lui présente ou lui rappelle la possibilité d'assurer son éternelle jeunesse, non dans l'attente, mais dans un fils de sa chair, l'élément de son éternité. Pour cela il lui faut se décider. Pourquoi pas avec celle qu'il a devant lui, sans avoir à chercher ailleurs ? Car après tout, lui dit-il,

16 Penser aux longues fiançailles imposées autrefois aux jeunes gens, souvent organisées par d'autres qu'eux, par les familles. 
Les voix de l'homme et de l'ombre dans

Quand cinq ans seront passés de F. García Lorca

« Ce n'est pas ton infidélité qui me gêne. Toi tu n'es rien. Toi tu ne signifies rien. C'est mon trésor perdu. C'est mon amour sans objet. Mais tu viendras!» (p. 271-272)

Il essaie de l'entraîner en affirmant : «Pour que je ne meure pas » (p. 272) En d'autres termes, il réalise que cette femme, qu'il a beaucoup rêvée mais qu'il ne semble pas désirer spécialement, aurait pu lui permettre d'assurer son devoir d'homme social et son éternité en procréant avec lui. Il ne se rend pas compte que dans ce duo la seule voix que l'on entende est celle de son moi qui surgit de l'ombre. Le Jeune Homme dévoile ainsi la face cachée de sa personnalité. Il ne pense qu'à lui. La face obscure de son égocentrisme est ainsi mise en évidence. Nous en avons la preuve quand le Jeune Homme interroge : "Que vais-je faire de cette heure qui vient et que je ne connais pas ? Où vais-je ? » (p. 276)

A cette question de son subconscient, la lumière baisse. Les ampoules portées par les anges prennent une teinte bleutée. La lumière de la lune entre par le balcon. Le bleu et la lune, les voix symboliques du rêve, éclairent la scène.

Un mannequin revêtu de la robe de la mariée qui était déjà prête entre. Le dialogue s'instaure entre le Jeune Homme et cet objet humanisé, en fait son antagoniste, qui se fait voix de l'obscur désir d'éternité. Elle est la voix de l'inconnue que le Jeune Homme recherche pour la revêtir de la robe symbolique.

«Mannequin : Qui utilisera le bel argent

de la brune et petite fiancée ?

Qui mettra ma robe? Qui la mettra ? »

(p. 277).

Et elle se plaint de n'avoir été brutalisée par personne :

« Je chante

Une mort que je n'ai pas connue,

la tristesse d'un voile jamais utilisé, avec un pleur de soie et de plume. » (p. 277)

Elle l'accuse :

«C'est de ta faute.

Tu aurais pu être pour moi poulain de plomb et d'écume, 
Tu aurais pu être hennissement et tu es lagune endormie... » (p. 280)

Elle lui présente un vêtement de nourrisson, ce qui va orienter la réflexion et la démarche du Jeune Homme.

Au Mannequin qui lui dit : « Mon fils. Je veux mon fils ! » il répond : « Oui, mon fils » (p. 283)

Il a enfin réalisé où se trouve sa voie. Elle lui redemande : «Qui mettra ma robe? Qui la mettra ?» (p. 284) et lui de répondre (p. 284)

«(Enthousiaste et ferme)

La mettra la femme qui attend au bord de la mer» (p. 284)

Mon enfant chante dans son berceau, et comme c'est un enfant de neige il attend aide et chaleur ». (p. 285)

Cependant un doute plane encore sur les résolutions du héros quand il parle d'un enfant de neige, car celle-ci, froide et blanche, est symbole de stérilité et de mort, symbole de la glaciation psychique et physique. Un enfant de neige est un enfant déjà avorté.

De toute façon, bien que le Vieillard, voix obscure de l'abstention, lui prône l'inaction : "Attends ! Attends ! ne m'abandonne pas je suis blessé ! Attends!» (p. 290). La voix de l'instinct le fait enfin sortir de son enfermement métaphysique, courir dans la rue chercher la réalisation de son souhait de procréation.

Mais, on l'aura remarqué, cette voix de l'instinct n'est que l'écho de son narcissisme et de son égoïsme. Ce qu'il va chercher, c'est une femme-objet à féconder, et non la femme qui l'aime et à qui il se donnera. C'est encore la voix obscure de l'égocentrisme qui aura étouffé celles de l'altruisme et de l'amour.

A ce point du drame, il faut noter que si l'acte II est le temps de cette évolution, celle-ci est déjà annoncée à l'acte I par les voix de l'inconscient ou du rêve qui se sont déjà faites 
entendre dans le récit second de la chatte et de l'enfant mort que nous avons signalé plus haut ${ }^{17}$. Évoquons-les.

Le jeune Homme vient de dire :

«Peu importe ce qui se passe dehors. Cette maison est à moi et personne n'entrera ici.

(On entend un coup de tonnerre lointain)

L'ami : Tous ceux qui voudront entrer entreront, non pas ici, mais sous ton lit. (Coup de tonnerre plus rapproché). »(p. 216)

A ce moment, alors que le Jeune Homme imagine, rêve dans l'espace diégétique fermé de sa bibliothèque, son débat avec ses doubles, il se crée, à un niveau second du rêve, un nouvel espace, hétérodiégétique, toujours onirique, mais lyrique cette fois, où la vie, paradoxalement représentée par deux êtres morts, entre dans le monde fermé du rêveur.

Voyons avec quelques clés de la symbolique lorquienne l'information didascalique qui introduit cette scène :

«On entend un autre coup de tonnerre (la tempête annoncée va envahir l'espace de tranquillité que le Jeune Homme veut protéger). La lumière (naturelle, du réel) baisse et une clarté bleue d'orage (le bleu symbole du rêve indique de quel espace il s'agit) envahit la scène. Les trois personnages se cachent derrière un paravent noir brodé d'étoiles. (Le théâtre sous le sable se substitue au Théâtre à l'air libre). Par la porte de gauche (la porte qui, du passé, du paradis perdu, ouvre sur l'inconscient) apparaissent l'Enfant Mort et le Chat. L'enfant est vêtu de blanc, en costume de premier communiant, avec une couronne de roses blanches sur la tête. Dans son visage de cire peinte, ressortent ses yeux et ses lèvres de lys desséché. Il porte le grand brassard de ruban à fleurs d'or et tient à la main un cierge torsadé. Tout indique un enfant très jeune).

"Le Chat est bleu avec deux énormes taches de sang rouge sur sa petite poitrine blanche/grise (le cœur) et sur la tête (l'âme). Ils avancent vers le public. L'enfant tient le chat par une patte. » (p. 217)

17 Voir plus haut, p. 5. 
De cette scène irréelle diverses interprétations sont possibles. Le Jeune Homme, face aux dangers que présente l'existence et aux difficultés de la vie, rêve le paradis perdu de son enfance. Il imagine l'enfant qui meurt à chaque instant en lui, qui chante avec lyrisme la liberté, la nature, la vie, et cherche le moyen d'éviter la porte de droite, la porte du réel, de la mort.

C'est ainsi que l'enfant entre en scène tenant par la patte un chat avec qui il parle, souvenir peut-être d'un jouet en peluche que le Jeune Homme, ou Lorca, devait avoir pour confident et qui se révèle alors n'être qu'un de ses nombreux doubles ${ }^{18}$.

Mais il représente aussi l'enfant qu'il désire et voudrait voir vivre, comme celui qui vit déjà dans la Fiancée qui l'a repoussé, alors que chez lui cet enfant est déjà avorté.

Pour la Chatte le chant est le même. Elle et l'Enfant seront obligés de disparaître de ce monde où ils jouaient (p. 221), où ils sont morts, la première par la méchanceté des enfants (des hommes) l'autre d'une crise cardiaque (p. 218) ou pour des raisons encore inexplicables.

D'autres interprétations sont possibles nous semble-t-il. La Chatte tuée par la méchanceté des hommes, ne serait-elle pas le double, la voix de la Dactylo blessée au cœur et à l'esprit par le jeune homme qui l'a rejetée ? L'enfant mort ne serait-il pas la voix de l'enfant que le héros a lui-même été et qu'il désirerait faire renaître de ses amours avec une femme qu'il recherchera à l'acte III ?

Transfert de symboles, glissement d'images, n'est-il pas enfin cet enfant mort qu'il a imaginé dans son rêve second, qui, dès qu'il apprend qu'il a affaire à une chatte et non un chat s'exerce au jeu de la séduction? :

"Chatte : Ne m'appelle plus Chat.

Chatte : Je suis une chatte.

Enfant : Non?

Chatte (coquette) : Tu aurais dû le savoir. Enfant : Comment ?

Chatte : Par ma voix d'argent. »

Et l'enfant de se faire déjà homme :

«Enfant (galant) : Tu ne veux pas t'asseoir ? Chatte: Si. J'ai faim. Enfant : je vais essayer de te trouver une souris. » (p. 219-220)

18 Cf. Nadine LY, Les Langues Néolatines, op. cit., p. 132. 
N'aurions-nous pas là une des voix profondes de l'homme, celle de l'élan vital, qui va le pousser à se compromettre, à séduire pour renaître dans son enfant, qui s'exprimait déjà d'une façon proleptique dans une scène imaginée par le rêveur ? Nous nous apercevons maintenant que si la scène détruisait la logique fictionnelle et la chronologie, elle annonçait l'avenir, et tout l'avenir de la pièce : l'enfant de neige, l'enfant mort-né.

Au début de l'acte III ler tableau, le rideau se lève sur un décor de forêt, métaphore du monde dans lequel l'homme cherche sa voie. Arlequin entre, vêtu de noir (symbole de deuil et de désespérance), et de vert (symbole d'espérance et de félicité). Fonction au double masque, il récite un poème sur le rêve et le temps.

Un nouvel acteur, la Jeune Fille, apparaît ; elle dit sa joie d'avoir retrouvé son fiancé :

$\begin{array}{ll}\text { «Jeune Fille : } & \text { Qui le dit } \\ & \text { Qui le dira ? } \\ & \text { Mon amant m'attend } \\ & \text { au fond de la mer. } \\ \text { Arlequin (moqueur) : } & \text { Mensonge. } \\ \text { Jeune Fille : } & \text { C'est la vérité. } \\ & \text { J'ai perdu mon désir, } \\ & \text { J'ai perdu mon dé, } \\ & \text { Et dans les grands bois } \\ & \text { Je les ai retrouvés. » }\end{array}$

(p. 294-295)

La Jeune Fille se présente ainsi comme le double du Jeune Homme qui a enfin réalisé la façon de satisfaire son instinct d'éternité ; nous l'avons vu plus haut. Et son comportement, malgré les apparences, n'a rien d'étrange lorsqu'Arlequin lui dit :

« Tu verras ton amant

au retour

du vent et de la mer. » (p. 296)

et qu'elle s'écrie «(effrayée) : Mensonge. » (p. 296)

En effet, à l'instar de son double, le Jeune Homme, elle est effrayée par l'éventualité d'une union qui se présente à elle mais qu'au fond d'elle-même elle ne souhaite pas non plus ... avant cinq ans, donc sans doute jamais. 
Elle s'annonce également comme le double prémonitoire de la Dactylo amoureuse dans une scène suivante qui, retrouvée et sollicitée par le Jeune Homme, rejettera à cinq ans son union avec lui. Celle-ci, héritière devenue du narcissisme de ce dernier, sera l'allégorie de leur refus de l'action et de leur égocentrisme. Et elle en châtiera le Jeune Homme tout en se châtiant elle-même.

Conscient de son échec, le Jeune Homme, à la fin du ler tableau, ne s'est pas encore rendu compte de ce que nous pensons être son erreur de comportement, c'est-à-dire son mépris de la femme et son désir de n'en faire qu'une femme-objet porteuse de son fils.

Quand les Parques, voix de l'Ombre, lui réclament sa carte, son as de cœur, il comprend. Mourant d'avoir rejeté l'idée d'aimer la Femme - l'amour hétérosexuel -, il se retrouve seul, même abandonné par son domestique discret mais omniprésent dans la pièce.

Vie, échec, solitude et mort, Quand cinq ans... n'est pas qu'une légende du temps. C'est aussi une légende de la désespérance d'un être en situation dans une société hantée par le spectre funeste de la mort.

Depuis le début de la pièce où le Jeune Homme entend un gémissement (p. 197), jusqu'à la fin du héros dont le fil de vie est coupé par Atropos (p. 197), la mort est évoquée successivement, à l'acte I par le bruit de vitres brisées (p. 215), par l'enfant de la concierge que l'on enterre (p. 232), par le chat mort (p. 231); le 2e Ami a vu un enfant qu'on allait enterrer, le même sans doute (p. 238); à l'acte II, le Mannequin évoque l'enfant dans son berceau mais qui est déjà un enfant de neige (p. 285) ; Acte III, la Femme en jaune vient d'abandonner le comte et son fils qu'il a laissé mourir (p. 306) ; dernier tableau, le Domestique et la Domestique donnent des informations sur l'enfant mort, le fils de la concierge (p. 232-233), avant que la mort mythique ne vienne emporter le Jeune Homme (p. 352) comme la chatte, comme l'enfant, comme toute l'humanité, malgré tous leurs efforts pour assurer leur éternité.

Devant une telle présence, métaphore d'une réalité sinistre et inéluctable, on peut se demander si toute la pièce n'est pas que la représentation d'un destin tragique déjà 
scellé. Nous aurions alors la représentation classique du théâtre grec ; l'argument connu, on en explique les passions qui l'ont généré. Le temps étant aboli dans le premier acte, on peut penser que le temps, pas plus que les actes I, II, et ler tableau du IIIe acte n'ont existé, et qu'ils ne sont que l'exposition d'une réalité que le spectateur ne comprendra qu'à posteriori, celle de la durée, même si, en définitive, cette dernière sera défaite par Chronos.

Nous avions noté déjà qu'au début de l'acte I comme à la fin il est toujours six heures. Le temps de la fiction n'a pas progressé ; mais celui de la narration lui, ne s'est pas arrêté. Nous avons vu alors se superposer au temps vécu du ler cyberacteur, le Jeune Homme, celui des trois autres personnages, l'Ami, le 2e Ami, le Vieillard. Tous doubles spéculaires rêvés par le héros et porteurs de leur propre sens, narrés donc par leur sémantisme. Or, les trois dialogues, les trois récits seconds dont ils sont porteurs, le temps du récit s'étant arrêté à 6 heures, ne se sont pas succédés dans le temps chronologique comme le laisserait entendre le continuum du récit premier, mais ils se sont superposés. A la linéarité objective de Chronos, à la fugacité de l'instant, s'est substituée une prise de conscience de l'épaisseur de la durée ${ }^{19}$ exprimée dans un triple dialogue du Jeune Homme avec lui-même.

C'est dans ces dialogues, qui reviennent à une véritable voix intérieure avec ses méandres, ses anachronies, ses atopies et l'absurdité existentielle qui le sous-tend, que le héros vit son drame et cherche à se réaliser.

La durée flotte sur le temps jusqu'au 2e tableau du $3 \mathrm{e}$ acte. Là, Chronos ne laissera pas la rêverie l'emporter, et la fin tragique est annoncée dans les vers analeptiques qui finissent le poème d'ouverture de l'acte III, porté par la voix d'Arlequin :

«Et si la rêverie feint d'élever des murs dans la plaine du Temps, le Temps lui fait croire qu'elle naît à cet instant.

19 J. ONIMUS : L'Expression du temps dans le roman. Revue de Littérature comparée (extrait) Paris, Didier, 12e année p. 229- 317. 
Aïe comme la nuit chante ! Comme elle chante !

Que de glaçons bleutés elle porte ! » (p. 294)

Nuit, glaçons, bleu, série de termes dont la narraticité éveille l'idée de mort tragique. Chronos et Thanatos se sont rejoints dans la pièce.

Si une raison de cette situation nous est déjà connue - le refus du Jeune Homme de se décider à l'action et de donner son cœur à la femme qui l'aime - il en existe d'autres qu'il ne veut pas donner "pour des raisons qui ne sont pas à expliquer. » (p. 196)

Le comportement du Jeune Homme vis-à-vis de la femme éclairera peut-être à ce sujet. Prenons des exemples.

$\mathrm{Au}$ premier acte, la Dactylo apparaît et une didascalie apprend qu'elle traverse la scène. Elle pleure (p. 194). A la fin de l'acte nous comprenons pourquoi ; quand elle reparaît elle porte une valise. Elle traverse à nouveau la scène et, au milieu, elle se retourne rapidement :

« Dactylo: Tu m'avais appelée?

Jeune Homme (fermant les yeux) : Non, je ne t'avais pas appelée.

(La Dactylo sort, le regard angoissé, espérant son appel). Dactylo (sur la porte) : As-tu besoin de moi ?

Jeune Homme (fermant les yeux) : Non. je n'ai pas besoin de toi.

La dactylo sort. » (p. 242-243)

Elle a été dirons-nous sèchement renvoyée. Ce comportement méprisant vis-à-vis de la femme employée se retrouve vis-à-vis de la femme future épousée. Le Jeune Homme devise avec le Vieillard et parle de la fiancée :

«Je l'ai peu connue. Mais pas d'importance. Je pense qu'elle m'aime. »

« Ils sont partis (son père et elle) pour un long voyage.

J'en étais presque heureux. " (p. 195)

On pourrait arguer des mariages préparés, des longues fiançailles traditionnelles de l'époque pour expliquer ce comportement; néanmoins la distance que veut mettre le Jeune Homme entre la femme et lui est importante et étrange. Il en est de même quand il déclare au Vieillard que le Père n'est jamais venu pour la visite protocolaire, et que celle-ci 
Les voix de l'homme et de l'ombre dans

Quand cinq ans seront passés de F. García Lorca

n'aura pas lieu tant que cinq ans ne seront pas passés (p. 196). Il semble que cela ne le dérange pas et qu'il ne soit pas pressé. Rappelons, ici, qu'au début de l'Acte I il lui disait : "Je gardais les bonbons pour les manger plus tard» (p. 192). Le lecteur averti sait que pour Freud, les bonbons symbolisent dans la représentation onirique les plaisirs érotiques. Il les repousse donc pour le moment ${ }^{20}$.

Observons maintenant le comportement du prétendant vis-à-vis de ses deux prétendues. Les pronoms qu'il utilise avec elles sont le Je, le Tu, quand il veut prendre et dominer. Le pluriel d'amour du couple ne l'est pas. A l'acte II comment le Jeune Homme s'adresse-t-il à sa fiancée ? :

«(Energique) : Je ne cherche pas. Moi, je t'ai. »

«Toi tu n'es rien. Tu ne signifie rien. » (p. 272)

Ce n'est pas elle qui l'intéresse mais l'enfant qui assurera son éternité.

A l'acte III il retrouve la Dactylo. Elle chante :

«Où vas-tu mon amour,

mon amour!»

Il lui répond :

«Où vas-tu, mon amour,

ma vie, mon amour...»(p. 313)

Là aussi, une fois encore il se trahit. Toute métaphore mise à part, il ne s'agit que de « sa vie » et non de « leur » amour.

Ce qui ressort de ces exemples, c'est une voix, celle de la mysoginie du Jeune Homme. Il y a de sa part, à l'encontre de la femme, une absence d'attirance physique. Quand il parle de l'amour il essaye de se leurrer, de se convaincre d'être ce qu'il n'est pas.

Si nous nous penchons maintenant sur les trois portraits de femme dans la pièce, notre impression se confirmera.

20 Sigmund FREUD. O.C. 3 tomos traducción de Luis Lopez Ballesteros. Edit. Biblioteca Nueva 4ed Madrid 1981, p. 2217, cité par Huélamo Kosma in "La influencia de Freud en el teatro de García lorca "Boletín de la Fundación Federico García Lorca, $\mathrm{N}^{\circ} 6$, diciembre de 1989. 
Telle que l'imagine le héros, la Fiancée est en quelque sorte le double féminin de la brute humaine robotisée qui la tient dans ses bras. Elle se révèle aussi mécanisée et fantastique que lui. Ses désirs charnels apparaissent sans aucune réserve. Sa voix dans son discours lyrique adressé à son centaure chante la victoire des sens sur la mesure et la décence. Ce que n'apprécie peut-être pas dans son subconscient le Jeune Homme effrayé par un tel comportement.

Le Mannequin, double de la Fiancée, de la Dactylo, du Jeune Homme même $e^{21}$, symbole de la femme à la recherche de celui qui l'aimera et la fécondera, reproche au héros son comportement pusillanime, son refus d'être en quelque sorte celui qui satisfera son désir et ses instincts profonds, celui qui aurait dû être un poulain de plomb et d'écume, un hennissement, et qui ne fut qu'une lagune endormie. Elle lui reproche donc son refus d'union charnelle, son refus de l'amour hétérosexuel.

La Femme en jaune, elle, est " un véritable enchantement » (p. 303). Elle est couverte de paillettes d'or, de faux or. Elle s'agite sur l'estrade, créant par le mouvement et ses propos délirants un espace atopique universel où elle situe sa problématique et rassemble les thèmes récurrents de la pièce : la femme abandonnée, l'enfant désiré, la détresse et la situation des personnages, même si en se les attribuant elle les inverse, transformant à son gré, dans ses affabulations, la vérité. La misère l'a poussée à se prostituer. Personnage insaisissable et sans doute effrayant, peut-être repoussant, que le Jeune Homme imagine et en qui il peut voir par glissement le reflet de certains aspects de sa propre personnalité.

Ces deux tendances qui dominent chez le héros, son désir de paternité et sa mysoginie sont contradictoires. Elles montrent la dualité de son être et la lutte que se livrent en lui la voix de l'Homme, social et anxieux de son éternité, et celle de l'Ombre, celle d'une identité profonde qui est la cause de son impuissance. Mais à l'époque il était impossible de parler de

21 Voir Nadine LY «Analyse d'un fragment de «Así que pasen cinco años : le duo du Jeune Homme et du Mannequin " in Le Théâtre impossible de Lorca. El Público. Así que pasen cinco años, Ramond Michèle Edit. Paris. Éd. du Temps, 1998, p. 19.40. 
cette voix de l'Ombre sur une scène « à l'air libre ». La magie de la poésie et la féerie du théâtre " sous le sable » ont permis au poète de se livrer et d'exprimer sincèrement le problème d'un exclu. Nous ne donnerons que quelques exemples :

Acte I : la Dactylo s'offre au héros qui la repousse. Il lui avoue : «Ce n'est pas moi qui te retiens. Tu sais bien que je ne peux rien faire. Je t'ai déjà dit quelquefois d'attendre... » (p. 207)

Au Vieillard il explique son comportement : «Je voudrais l'aimer comme je voudrais avoir soif devant les fontaines. Je voudrais... » (p. 209)

Mais, ne rien pouvoir faire avec une femme, désirer avoir soif devant cette fontaine, c'est bien avouer déjà une soif de désir, et une impuissance à la satisfaire. La suite confirme cette impression.

L'Ami entre et après l'avoir pris dans ses bras et l'avoir fait tournoyer, il lui demande, nous nous en souvenons : «Et où sont dans cette maison les portraits des filles avec qui tu couches ? (p. 210)

Un moment après il joue avec lui, il frotte son nez contre le sien, le chatouille, le Jeune Homme réagit : il rit. Ils luttent, l'Ami lui prend la tête entre les jambes et le tape (p. 214).

Si ce jeu est clair dans le cadre d'un amour hétérosexuel, il peut l'être également dans celui d'un amour homosexuel.

Avec le Mannequin nous avons vu plus haut que cette vision de la fiancée telle que le héros a pu la percevoir, dédoublée dans un mannequin de luxe sollicitée par les sens et l'instinct, peut être signe de mysoginie ; elle n'est pas que cela. En effet, quand le Mannequin à l'acte II clame sa désespérance et dit :

« Je chante

la mort que je n'ai pas connue

Qui portera la jolie robe

de la petite fiancée brune ? » (p. 277-278)

A cette complainte sur l'amour qu'elle n'a jamais eu et à cette question qu'elle adresse au Jeune Homme, celui-ci répond :

«Personne ne portera ta robe, forme blanche et vague lumière, 
car la soie et le givre ont bâti

de fragiles architectures. » (p. 278)

La réponse est nette. Sa tentative, il le sent, ne ressort que du rêve. Il ne se décidera jamais à "sauter le mur » (saltar el muro) (p. 335), à déclarer son désir à une femme avec conviction, ne serait-ce que pour assurer son éternité ; il s'en sait incapable; car, comme le lui signale la Dactylo à l'acte III, ler tableau, lors de son ultime tentative : «Toi tu n'as pas d'yeux pour me voir nue, ni de bouche pour embrasser mon corps infini. Laisse-moi. » (p. 324)

La dactylo lui jette au visage son incapacité à réagir en homme, son malaise, son impuissance devant la femme, sa vérité donc, et à son tour elle le repousse.

Alors un personnage va prendre tout son sens. Dans une œuvre où le héros semble seul22 à créer des êtres de fantaisie, des allégories qui miment les méandres de son monologue intérieur et toute la problématique de l'œuvre, un être personnifié - il a un prénom - prend tout son relief, et sa voix muette surgit de l'ombre pour crier du fond du subconscient la vérité du Jeune Homme, du poète, de la pièce : c'est Juan, le domestique.

Certains critiques ont vu en lui le représentant du peuple des humbles cher à Lorca, d'autres le destin. Nous y verrons la voix de l'interdit qui demande à ne plus l'être.

$\mathrm{Au}$ début de l'acte I une didascalie. " un domestique subtil qui marche sur la pointe des pieds ferme la fenêtre» (p. 198).

22 Nous posons ici le problème de l'existence ou non du ou des personnages.

Le Jeune Homme au moment de mourir se retrouve seul. On peut penser qu'il est le seul personnage réel de la pièce. Nous voudrions faire remarquer que par l'impact du théâtre il est aussi un cyberacteur et que par lui toutes les informations, tout le message de la pièce ont été transmis à un autre acteur, narrataire de la fiction : le spectateur. Celui-ci en effet, signalons-le, est interpellé par le clown à l'acte III. Il entre donc dans l'espace théâtral comme acteur et partenaire direct, et lui aussi a pour rôle de comprendre la pièce, de comprendre le personnage principal. Pourquoi ne pas penser que ce personnage principal n'existe guère de lui-même, et que le poète ne le fait exister que dans la recréation que fait de lui chaque spectateur-narrataire, acteur de ce théâtre du monde. Il existe, sans doute, mais en particulier dans chacun de ceux qui comprendront et vivront son drame, celui de Lorca. 
Le héros ne veut pas que les bruits de la rue entrent chez lui. A moins, penserons-nous, avec certaines critiques, qu'il ne veuille pas que les bruits de chez lui parviennent dans la rue et que sa vérité soit connue.

Le domestique est là quand, au dernier tableau, le Jeune Homme entre dans la bibliothèque "donnant des signes de désespérance et de faiblesse physique » et dit à son valet : «Donne-moi un verre d'eau fraîche. » (p. 334)

Juan le sert. Nous pensons que le signe est clair en ce qui concerne la relation qui unit les deux personnages. Nous avons eu le même, en contrepoint, quand le Jeune Homme déclarait au Vieillard, au sujet de la Dactylo amoureuse de lui : "Je voudrais l'aimer comme je voudrais avoir soif devant les fontaines. » (p. 209)

Il refusait de boire à la fontaine de la Dactylo, il boit à celle de Juan.

Cette soif de l'amour d'un homme, le héros la clame au moment où, en ses derniers instants, alors que sonnent les douze coups de minuit, il se retrouve seul et il crie son désir : «Il n'y a aucun homme ici ? » (p. 353) ${ }^{23}$

Cette phrase peut être interprétée différemment, il est remarquable pourtant que lui mort, le valet apparaisse avec un candélabre, symbole de la lumière spirituelle, de semence de vie et de salut.

Nous nous sentons bien incompétent pour avancer toute analyse psychanalytique ${ }^{24}$. Mais peut-être avons-nous

23 « ¿ Ningún hombre aquí ? " Cet appel est présent dans Teatro contemporáneo. Teatro inquieto español, Madrid, Aguilar, 1967, p. 341. Il se trouve en note dans l'édition de référence (note 76, p. 352-353.) Voir à ce sujet l'article de Eutimio Martín, in Le théâtre impossible de Lorca... op. cit., p. 141-155. Il relève du reste la déclaration de Lorca dans une de ses lettres à Rafael Martinez Nadal, où il exprime sa satisfaction d'avoir écrit un drame "sur un thème franchement homosexuel ». Il s'agit de El Público, et E. Martin commente : «El Público es francamente homosexual. Así que pasen cinco años lo es cripticamente. Hay que desvelarlo » p. 150-151. (Le Public est franchement homosexuel, Quand cinq ans seront passés : Il faut déchiffrer le cryptogramme.)

Nous avons opté pour cette interprétation des dernières répliques.

24 Voir sous cet aspect l'approche de Jocelyne Aube Bourligueux : «Aux sources du refus, des fantasmes et de l'aliénation chez le 
là un appel à ce qu'apparaisse la vérité. A ce qu'apparaisse au grand jour les pulsions secrètes qui sont vie. N'est-ce pas là un appel du poète Lorca à ce que les voix de l'Homme et de l'Ombre puissent s'exprimer ensemble aux yeux du monde ? Et les douze coups de minuit ne seraient-ils pas un mur dans la plaine du temps marquant la fin d'une époque d'ostracisme et annonçant l'avènement d'un jour nouveau où le droit à la différence serait reconnu ? où la voix de l'Ombre pourrait devenir aussi une voix de l'Homme?

\footnotetext{
"Jeune Homme » de "Lorsque cinq ans seront passés " de F. García Lorca : l'enfant et le chat ". Littérature, médecine, société, Université de Nantes, $n^{\circ}$ 5, 1983 (Fantasmes et folies) p. 209-238.
} 